\title{
Negative hydrogen ion dynamics inside the plasma volume of a linear device: estimates from Particle-In-Cell calculations.
}

\author{
G. Fubiani, ${ }^{1, a)}$ R. Agnello, ${ }^{2}$ I. Furno, ${ }^{2}$ L. Garrigues, ${ }^{1}$ Ph. Guittienne, ${ }^{2}$ G. Hagelaar, ${ }^{1}$ A. Howling, ${ }^{2}$ R. Jacquier, ${ }^{2}$ \\ B. Reman, ${ }^{1}$ A. Simonin, ${ }^{3}$ and F. Taccogna ${ }^{4}$ \\ 1) LAPLACE, Université de Toulouse, CNRS, Toulouse, France \\ ${ }^{2)}$ Ecole Polytechnique Fédérale de Lausanne (EPFL), Swiss Plasma Center (SPC), CH-1015 Lausanne, \\ Switzerland \\ 3) CEA, IRFM, F-13108 St Paul lez Durance, France \\ ${ }^{4)}$ CNR-Institute for Plasma Science and Technology, Bari, Italy
}

Negative hydrogen or deuterium ions are the precursor particles used to generate a high power beam of neutrals in order to heat the tokamak plasma core of magnetic fusion devices, inject current and to some extent control instabilities. In the case of ITER for instance, the negative ions are produced inside a high power large volume low pressure tandem type magnetised ion source and extracted toward an electrostatic accelerator which accelerates them to $1 \mathrm{MeV}$ before entering a neutraliser converting the ions into a neutral beam. This so-called Neutral Beam Injector (NBI) relies on the production of negative ions on the surface facing the plasma of the ion source extraction electrode. The latter is covered by a cesium layer in order to increase the negative ion yield. The use of cesium is currently an issue as it may diffuse outside of the source and induce secondary particle production or voltage breakdowns inside the accelerator vessel requiring a regular maintenance in a nuclear environment. In this work, we analyse numerically with a $2.5 \mathrm{D}$ ParticleIn-Cell model the production rate and transport of negative ions in a linear device used as an ion source. The negative ions are generated via a dissociative attachement process with a hydrogen molecule in the volume of a magnetised cesium-free plasma. The linear device in the model has a large aspect ratio with a radius of $5 \mathrm{~cm}$ and a length of $100 \mathrm{~cm}$ and the magnetic field strength ranges from 100 to 400G. We show that the shape and depth of the plasma potential profile may be controlled by biasing the end-plates which in turn strongly influence the residence time of the electrons and hence the negative ion yield. We observe the formation of large scale rotating structures when the positive ions become magnetised with a rotation velocity in the $\mathrm{kHz}$ range.

\section{INTRODUCTION}

Negative hydrogen isotope ions may be produced either by the dissociative attachment of a vibrationally excited hydrogen molecule or via the tunnelling process of electrons from a metal conduction band toward a neutral atom or positive ion impacting its surface. The former is a two step process where one region of the plasma source has typically a higher electron temperature of about 5-10 $\mathrm{eV}$ in order to excite vibrationally the hydrogen molecule by electron impacts, $\mathrm{e}+\mathrm{H}_{2}\left(\nu_{\mathrm{i}}\right) \rightarrow \mathrm{e}+\mathrm{H}_{2}\left(\nu_{\mathrm{j}}\right)$ where $j>i$ and $\nu$ is the vibrational quantum state of the molecule, while in a second area the electron temperature must drop to $\sim 1 \mathrm{eV}$ to optimally produce negative ions by a dissociative attachment process ${ }^{1,2}$, e.g., $\mathrm{e}+\mathrm{H}_{2}\left(\nu_{\mathrm{j}}\right) \rightarrow \mathrm{H}^{-}+\mathrm{H}$. A low electron temperature region also lengthens the mean-free-path of a given negative ion before being destroyed by collisions with electrons or the background gas. In practice, a plasma source with a strong gradient in electron temperature is generated by implementing a magnetic filter field between the discharge (region where the external power is coupled to the plasma) and the extraction region such as to decrease the mobility of the magnetised electrons and induce a local loss of electron kinetic energy by inelastic

\footnotetext{
a) Electronic mail: gwenael.fubiani@laplace.univ-tlse.fr
}

collisions. The magnetic field is transverse to the discharge axis. Another way to efficiently produce negative ions is through the interaction of atoms or positive ions with metallic walls. In high brightness ion sources, cesium is added in order to decrease the metal surface work function and hence increase the production yield of negative ions. Cesium is one component of negative ion sources used for fusion applications ${ }^{3}$ as well as in high energy linear particle accelerators ${ }^{4-6}$, devices used for neutron generation ${ }^{7}$, tandem accelerators and accelerator based mass spectrometry. ${ }^{8,9}$ In the case of fusiontype plasma sources, the extracted negative ion current produced by volume processes is only a small fraction of the total $(<10-20 \%)$; most ions originating from the surface of the extracting electrode. ${ }^{10}$ Cesium is currently an issue in Neutral Beam Injector (NBI) concepts as it typically diffuses outside of the ion source across the extraction apertures. In the accelerator vessel, cesium can induce voltage breakdowns, possibly negative ion beamlet halos or the production of secondary particles which will absorb power from the power supplies and can deposit a power density on the accelerator parts exceeding the damaging threshold for the material. ${ }^{11}$ Cesium hence requires a regular maintenance of the injector in a nuclear environment. The estimates for ITER and DEMO provide an evaporation rate of about $10 \mathrm{mg} / \mathrm{h}$ per oven in the ion source. ${ }^{3,12}$ As a consequence, there is currently a research effort in the low temperature plasma community to find alternatives to cesium either by studying other low 
work function materials ${ }^{13}$ or different ion source configurations providing a plasma confinement optimal for producing negative hydrogen isotope ions through volume processes in a cesium free plasma. One such candidate is a linear device and recent experiments on the Resonant Antenna Ion Device (RAID) ${ }^{14}$ at the Swiss Plasma Center (SPC) in Ecole Polytechnique Fédérale de Lausanne (EPFL) has shown the capability to produce a maximum negative ion density of $\sim 2 \times 10^{16} \mathrm{~m}^{-3}$ measured in both hydrogen and deuterium at a radius of 6 $\mathrm{cm}$ for a Radio-Frequency (RF) power (provided by a helicon antenna) of $5 \mathrm{~kW} \cdot{ }^{15,16}$ The peak electron density is about $2 \times 10^{18} \mathrm{~m}^{-3}$ and the background gas pressure $0.3 \mathrm{~Pa}$. The RAID testbed is a cylinder of $20 \mathrm{~cm}$ radius and $1.8 \mathrm{~m}$ length with a magnetic field strength aligned with the axis of up to $800 \mathrm{G}$. The volume of the source is $\sim 0.2 \mathrm{~m}^{3}$ and $5 \mathrm{~kW}$ is hence equivalent to a power density of $\sim 25 \mathrm{~kW} / \mathrm{m}^{3}$ (which is non-uniformly distributed). In comparison, the BATMAN one driver ITER-prototype ion source generates a line-of-sight (LOS) averaged negative ion density of $7 \times 10^{16} \mathrm{~m}^{-3}$ in the presence of cesium measured by Cavity Ring Down Spectroscopy (CRDS) at $0.45 \mathrm{~Pa}$ in hydrogen about $2 \mathrm{~cm}$ from the extraction electrode (so-called "plasma grid") for a power density of $\sim 1 \mathrm{MW} / \mathrm{m}^{3}$ (60 kW of RF power and a plasma volume of $\sim 50$ litres). ${ }^{17}$ Helicons are more efficient in terms of plasma production but the difference in power densities between the two concepts is also due to the increased plasma confinement in the case of a linear device (this in principle can favour the production of negative ions in the plasma volume). Electrons are strongly magnetized in the latter, RF power is optimally absorbed near the discharge axis (which is aligned with the magnetic field vector) and the $\mathbf{E} \times \mathbf{B}$ and diamagnetic drifts are closed (i.e., in the azimuthal direction). Losses consequently occur mainly on the surfaces short-circuiting the magnetic field lines. Note that the distance traveled by electrons along the field lines is typically of meter scale due to the large aspect ratio of these devices. Furthermore, in a tandem-type fusion-prototype device such as BATMAN or ELISE, the negative ions are produced on the cesium covered plasma grid (PG) surface, i.e., in the direct vicinity of the extraction apertures, and hence can be efficiently extracted toward the accelerator vessel. In a linear device, the negative ion density typically peaks near the edges of the region where the helicon antenna power is coupled to the plasma. The question of an optimal extraction of the negative ions remains an open problem at the present time; this likely requires a helicon antenna radius similar to the one of the linear device to shift the negative ion density maximum toward the cylinder side wall.

In this work, we analyse the production yield and transport dynamics of negative ions in a linear device using a 2-dimensional (2D) Particle-In-Cell model including losses in the third dimension calculated analytically (method commonly dubbed "2.5D" for that reason). We show that the residence time of the magnetised electrons can be significantly increased by biasing both end-plate electrodes with the same voltage. The latter are perpendicular to the magnetic field lines (which are straight) and hence a bias voltage may be used to control the electron losses along these lines. The residence time of the electrons is strongly correlated with the yield for producing negative ions in the plasma volume via the two step process consisting of the vibrational excitation followed by the dissociative attachement of the hydrogen isotope molecules. In addition, we observe the formation of large scale rotating structures at frequencies of about tens of $\mathrm{kHz}$ when the positive ions become somewhat magnetised. In the next section, we describe in detail the numerical model, the plasma chemistry which we implemented, and the simulation domain. In Sec. III, we analyse the plasma properties when the end-plates are biased negatively so as to reduce electron losses and hence increase their confinement. In Sec. IV, we compare the model predictions to the experimental measurements on the RAID device. In Sec. V, we study the impact of the magnetic field strength on the plasma dynamics and notably the appearance of instabilities. Lastly in Sec. VI, we discuss the possibility of extracting negative ions from a slit aperture embedded on the cylinder wall of the linear device. The numerical resolution does not allow us to model the details of negative ion extraction across the aperture. We analyse instead the effect on the 2D plasma potential profile of biasing positively an electrode on the wall; the aim being to assess whether or not one can channel negative ions from inside the plasma volume toward the aperture.

\section{NUMERICAL MODEL}

The numerical model is an explicit electrostatic Particle-In-Cell (PIC) algorithm with Monte-Carlo Collisions (MCC). Maxwell's equations are solved in the plasma volume within the electrostatic limit with the plasma potential derived from Poisson's equation. The magnetic field is provided by electromagnets and its profile is prescribed in the model. The algorithm is explicit and as a consequence, the source term for Poisson's equation (the total charge density $\rho$ ) is calculated from the particle trajectories estimated at the previous time step. We assume that the magnetic field lines are straight, parallel to the linear device axis (see fig. 1). The field strength $B_{0}$ is constant. The simulation domain and hence the numerical mesh are 2-dimensional (2D) with particle losses in the third dimension calculated analytically to account for a $3 \mathrm{D}$ volume. ${ }^{18}$ In that direction, the rates of positive ion losses are estimated from the Bohm velocity which is the average ion velocity at the entrance of the plasma sheath. The loss frequency is calculated as follows,

$$
\nu_{L}=2 \kappa u_{B} / L_{z},
$$


where $u_{B}=\sqrt{e T_{e}(x, y) / m_{i}}$ is the local Bohm velocity, $T_{e}$ the electron temperature, $L_{z}$ the length of the linear device and $\kappa=n_{s} / \bar{n}$ the ratio between the plasma density at the sheath edge $n_{s}$ and the average plasma density $\bar{n}$ along $(\mathrm{Oz})$. Equation (1) was derived by volume averaging the continuity equation in the $1 \mathrm{D}$ limit assuming quasi-neutrality. $\kappa \simeq 0.5$ in a low pressure plasma with ambipolar diffusion (along the magnetic field lines) and nearly collisionless ions. ${ }^{19}$ In this work the negative ion to electron density ratio is at maximum around $10 \%$ and the negative ion average kinetic energy $\sim 0.3 \mathrm{eV}$. For this reason, we considered that this ion specie has hence a minor impact on the plasma potential profile and Bohm velocity at the sheath entrance. ${ }^{19}$ The loss rate for the electrons is evaluated by following their trajectories in the third direction. The latter gyrate around the magnetic field lines (electrons are strongly magnetized in our case) along $(\mathrm{Oz})$ and are lost to the wall if their kinetic energies exceed the difference between the wall and plasma potentials $\frac{1}{2} m_{e} v_{z}^{2} \geq e\left(\phi-V_{e p}\right)$, where $m_{e}$ and $e$ are the electron mass and charge, $v_{z}$ its velocity in the z-direction, $V_{e p}$ the end-plate bias voltage and $\phi(x, y)$ the plasma potential calculated on the $2 \mathrm{D}$ mesh, respectively. In $2.5 \mathrm{D}$, the plasma parameters are consequently averaged values over the length $L_{z}$.

Electrons absorb a given power which is an external parameter to the model. We assume that a fraction $N_{h}=$ $N_{e} \nu_{h} \Delta t$ of the total number of macro-electrons $N_{e}$ in the power absorption region will couple the external power to the plasma during a time lag $\Delta t$ and furthermore that the absorbed energy per electron is identical on average, i.e., $T_{h}=\frac{2}{3}\left\langle\Delta E_{h}\right\rangle$ with,

$$
\left\langle\Delta E_{h}\right\rangle=\frac{P_{a b s} \Delta t}{N_{m} N_{h}},
$$

where we defined an artificial heating temperature $T_{h}$. $N_{m}$ is the macroparticle weight (the number of real electrons per macro-electron) and $\nu_{h}$ a heating frequency (which is arbitrary), respectively. We assume that the power absorption profile is Gaussian and the macroelectrons have hence a probability

$$
p_{c}=\exp \left[\frac{-\left(x_{i}^{2}+y_{i}^{2}\right)}{2 \sigma_{h}^{2}}\right]
$$

of undergoing a heating collision. $x_{i}$ and $y_{i}$ are the macro-particle coordinates and $\sigma_{h}$ is the standard deviation, respectively. Their velocity increments $\Delta v_{x}, \Delta v_{y}$ and $\Delta v_{z}$ are sampled from a Maxwellian distribution with a temperature $T_{h}$ such that,

$$
v_{i}^{\prime}=v_{i}+\Delta v_{i},
$$

and $i=x, y, z$. In practice, one loops over the $N_{e}$ macroelectrons inside the power absorption region. A candidate electron is selected randomly inside the particle array and the actual occurence of a collision is verified via

\begin{tabular}{|c|c|c|}
\hline$\#$ & Reaction & Cross section ref. \\
\hline 1 & $\mathrm{e}+\mathrm{H} \rightarrow \mathrm{e}+\mathrm{H}$ (elastic) & $20-24$ \\
\hline 2 & $\mathrm{e}+\mathrm{H} \rightarrow \mathrm{e}+\mathrm{H}$ (inelastic, 4 proc.) & 25 \\
\hline 3 & $\mathrm{e}+\mathrm{H} \rightarrow 2 \mathrm{e}+\mathrm{H}^{+}$ & 25 \\
\hline 4 & $\mathrm{e}+\mathrm{H}_{2} \rightarrow \mathrm{e}+\mathrm{H}_{2}$ (elastic) & 26 \\
\hline 5 & $\mathrm{e}+\mathrm{H}_{2} \rightarrow 2 \mathrm{e}+\mathrm{H}_{2}^{+}$ & 25 \\
\hline 6 & $\mathrm{e}+\mathrm{H}_{2} \rightarrow 2 \mathrm{e}+\mathrm{H}^{+}+\mathrm{H}$ (2 proc. $)$ & 25 \\
\hline 7 & $\mathrm{e}+\mathrm{H}_{2} \rightarrow \mathrm{e}+\mathrm{H}_{2}$ (inelastic, 16 proc.) & $25,27-32$ \\
\hline 8 & $\mathrm{e}+\mathrm{H}_{2} \rightarrow \mathrm{e}+2 \mathrm{H}$ (3 proc. $)$ & 25,33 \\
\hline 9 & $\mathrm{e}+\mathrm{H}_{3}^{+} \rightarrow 3 \mathrm{H}$ & 25 \\
\hline 10 & $\mathrm{e}+\mathrm{H}_{3}^{+} \rightarrow \mathrm{H}+\mathrm{H}_{2}$ & 25 \\
\hline 11 & $\mathrm{e}+\mathrm{H}_{3}^{+} \rightarrow \mathrm{e}+\mathrm{H}^{+}+2 \mathrm{H}$ & 25 \\
\hline 12 & $\mathrm{e}+\mathrm{H}_{3}^{+} \rightarrow \mathrm{e}+\mathrm{H}^{+}+\mathrm{H}_{2}$ & 25 \\
\hline 13 & $\mathrm{e}+\mathrm{H}_{2}^{+} \rightarrow 2 \mathrm{H}$ & 25 \\
\hline 14 & $\mathrm{e}+\mathrm{H}_{2}^{+} \rightarrow \mathrm{e}+\mathrm{H}^{+}+\mathrm{H}(2$ proc. $)$ & 25,33 \\
\hline 15 & $\mathrm{e}+\mathrm{H}_{2}^{+} \rightarrow 2 \mathrm{e}+2 \mathrm{H}^{+}$ & 33 \\
\hline 16 & $\mathrm{e}+\mathrm{H}^{-} \rightarrow 2 \mathrm{e}+\mathrm{H}$ & 25 \\
\hline 17 & $\mathrm{e}+\mathrm{H}_{2}^{*} \rightarrow \mathrm{H}^{-}+\mathrm{H}$ & 33 \\
\hline 18 & $\mathrm{e}+\mathrm{H}_{2}^{+} \rightarrow \mathrm{e}+\mathrm{H}_{2}^{+}$ & $(\text {Coulomb })^{34}$ \\
\hline 19 & $\mathrm{e}+\mathrm{H}^{+} \rightarrow \mathrm{e}+\mathrm{H}^{+}$ & $(\text {Coulomb })^{34}$ \\
\hline 20 & $\mathrm{e}+\mathrm{H}_{3}^{+} \rightarrow \mathrm{e}+\mathrm{H}_{3}^{+}$ & $(\text {Coulomb })^{34}$ \\
\hline
\end{tabular}
an additional random number $r$ between 0 and 1 such
TABLE I. Electron collisions.

that $r \leq p_{c}\left(x_{i}, y_{i}\right)$. The process is repeated until $N_{h}$ collisions have materialized. Equation (4) preserves the average velocity of the electron distribution function, that is, $\left\langle v_{i}^{\prime}\right\rangle=\left\langle v_{i}\right\rangle$. On average, the electron energy is increased by $\frac{1}{2} m_{e}\left\langle\Delta v_{i}^{2}\right\rangle$. Note that one can add a velocity increment along a single direction instead to mimic the power absorbed on average by an electron interacting with the RF wave generated by a time varying current in a coil. This method generates a Maxwellian Electron Energy Distribution Function (EEDF) in hydrogen (see sec. III B).

The complex physical-chemistry resulting from the interaction between electrons, ions and the hydrogen gas are included in the model. The neutral dynamics are not simulated and we implement a uniform gas density and temperature profile instead with a given background density $n_{\mathrm{H} 2}$ for the hydrogen molecules and $n_{\mathrm{H}}$ for the atoms corresponding to a dissociation rate $n_{\mathrm{H}} / n_{\mathrm{H} 2} \sim 0.25$ (which is typically observed in ITER-like ion sources. ${ }^{18}$ ) Collisions are modelled between charged particles and neutrals via the Monte-Carlo (MC) technique where we derive a velocity for the latter from a Maxwellian distribution at a temperature $T_{n}$ while for the interaction between electrons and positive ions (Coulomb collisions), the actual velocities of both the incident and target macroparticles are considered during the scattering process. ${ }^{35,36}$ The probability for the occurence of a reaction is calculated from the tabulated cross-sections and particles are scattered in their center of mass after the collision. For more details, please see ref. ${ }^{18}$. The set of re- 
TABLE II. Heavy particle processes.

\begin{tabular}{rll}
\hline \hline$\#$ & Reaction & Cross section ref. \\
\hline 1 & $\mathrm{H}_{3}^{+}+\mathrm{H}_{2} \rightarrow \mathrm{H}_{3}^{+}+\mathrm{H}_{2}$ (elastic) & 43 \\
2 & $\mathrm{H}_{3}^{+}+\mathrm{H} \rightarrow \mathrm{H}_{3}^{+}+\mathrm{H}$ (elastic) & 42,43 \\
3 & $\mathrm{H}_{2}^{+}+\mathrm{H}_{2} \rightarrow \mathrm{H}_{3}^{+}+\mathrm{H}$ & 43 \\
4 & $\mathrm{H}_{2}^{+}+\mathrm{H}_{2} \rightarrow \mathrm{H}_{2}+\mathrm{H}_{2}^{+}$ & 44 \\
5 & $\mathrm{H}_{2}^{+}+\mathrm{H} \rightarrow \mathrm{H}_{2}^{+}+\mathrm{H}$ (elastic) & 45 \\
6 & $\mathrm{H}^{+}+\mathrm{H} \rightarrow \mathrm{H}+\mathrm{H}^{+}$ & 45 \\
7 & $\mathrm{H}^{+}+\mathrm{H} \rightarrow \mathrm{H}^{+}+\mathrm{H}$ (elastic) \\
8 & $\mathrm{H}^{+}+\mathrm{H}_{2} \rightarrow \mathrm{H}^{+}+\mathrm{H}_{2}$ (elastic) & 25 \\
9 & $\mathrm{H}^{+}+\mathrm{H}_{2} \rightarrow \mathrm{H}^{+}+\mathrm{H}_{2}$ (inelastic, 4 proc.) \\
10 & $\mathrm{H}^{-}+\mathrm{H} \rightarrow \mathrm{e}+2 \mathrm{H}$ \\
11 & $\mathrm{H}^{-}+\mathrm{H} \rightarrow \mathrm{e}+\mathrm{H}_{2}$ & 42 \\
12 & $\mathrm{H}^{-}+\mathrm{H}_{2} \rightarrow \mathrm{H}^{-}+\mathrm{H}_{2}$ (elastic) \\
13 & $\mathrm{H}^{-}+\mathrm{H} \rightarrow \mathrm{H}^{-}+\mathrm{H}$ (elastic) \\
14 & $\mathrm{H}^{+}+\mathrm{H}^{-} \rightarrow 2 \mathrm{H}(2$ proc.) \\
15 & $\mathrm{H}^{+}+\mathrm{H}^{-} \rightarrow \mathrm{H}_{2}^{+}+\mathrm{e}$ \\
16 & $\mathrm{H}^{-}+\mathrm{H}_{2} \rightarrow \mathrm{H}_{2}+\mathrm{H}+$ e & 25 \\
17 & $\mathrm{H}^{-}+\mathrm{H}^{+} \rightarrow+\mathrm{H}^{-}$ & 25 \\
\hline \hline
\end{tabular}

actions is presented in tables I and II. ${ }^{18,37,38}$ Table I corresponds to the collision processes associated with electrons. Reactions \#2, 6, 7, 8 and 14 combine inelastic processes. Reaction \#2 regroups the excitation of the hydrogen atom from the ground state to the electronic level $n=2-5 .^{25}$ Reaction \#7 combines the ground state excitation of the hydrogen molecule $\mathrm{H}_{2}\left(\mathrm{X}^{1} \Sigma_{\mathrm{g}}^{+} ; \nu=0\right)$ to the vibrational levels $\nu^{\prime}=1-3^{25,31}$, electronic levels (for all $\nu^{\prime}$ ) $\mathrm{B}^{1} \Sigma_{\mathrm{u}}, \mathrm{B}^{\prime 1} \Sigma_{\mathrm{u}}, \mathrm{B}^{\prime \prime}{ }^{1} \Sigma_{\mathrm{u}}, \mathrm{C}^{1} \Pi_{\mathrm{u}}, \mathrm{D}^{1} \Pi_{\mathrm{u}}, \mathrm{D}^{\prime 1} \Pi_{\mathrm{u}}$, $\mathrm{a}^{3} \Sigma_{\mathrm{g}}^{+}, \mathrm{c}^{3} \Pi_{\mathrm{u}}, \mathrm{d}^{3} \Pi_{\mathrm{u}}{ }^{25}$, Rydberg states ${ }^{32}$ and lastly rotational levels $J=2^{27,28}$ and $3 .^{29,30}$ Reaction \#17 models the generation of negative ions in the ion source volume, which are a byproduct of the dissociative impact between an electron and molecular hydrogen $\mathrm{H}_{2}(\nu \geq 4) .{ }^{25}$ We do not calculate self-consistently the concentration of excited species in the model. To estimate the volume production of negative ions, we assume that $1.5 \%$ of $\mathrm{H}_{2}$ molecules (except in sec. IV) are excited in vibrational levels $\nu \geq 4$. This is in accordance with the $\mathrm{H}_{2}$ vibrational distribution function calculated with the $0 \mathrm{D}$ model of ref. ${ }^{39}$. Changing this parameter modulates the magnitude of the negative ion density. Table II summarizes the collision processes of heavy ions with neutrals. Reaction \#9 corresponds to the excitation of the hydrogen molecule from the ground state to vibrationally excited levels $\nu^{\prime}=1-2^{40,41}$ and to the rotational levels $J=2-3 .^{42}$ To our knowledge there are no reliable data available for the elastic collision between $\mathrm{H}_{3}^{+}$and neutral atoms (reaction \#2), we consequently use the same cross-section as in reaction \#1.

The dimensions of the grid must be of the order of the Debye length and one needs to resolve the electron plasma frequency in a PIC-MCC model otherwise

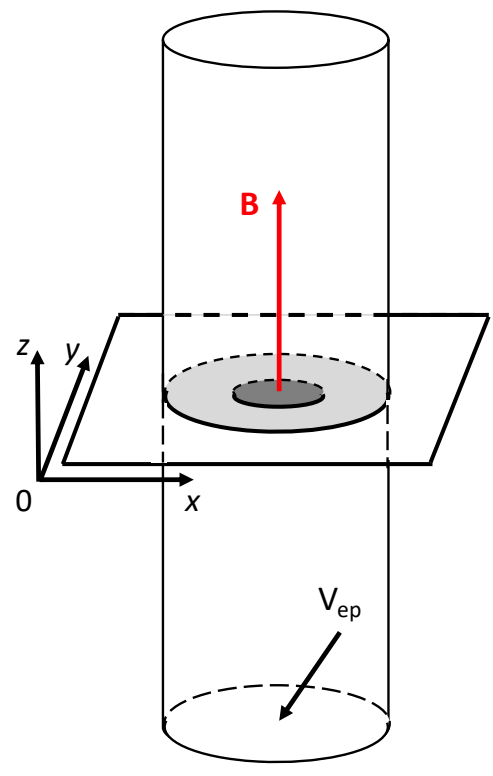

FIG. 1. Schematic illustration of the simulation domain. The numerical mesh is $2 \mathrm{D}$ and in the $(\mathrm{Ox}),(\mathrm{Oy})$ directions with particles losses along $(\mathrm{Oz})$ estimated semi-analytically via (1) the Bohm frequency for the positive ions while (2) trajectories of negative charges are followed with losses occurring if their kinetic energy in the z-direction exceeds the difference between the plasma and end-plate $\left(V_{e p}\right)$ potentials. In all the calculations except for sec. IV (comparison with the experiments), the radius of the linear device is $R=5 \mathrm{~cm}$, length $L_{z}=100 \mathrm{~cm}$, an absorbed power of $2.4 \mathrm{~kW}$ and a standard deviation for the power profile $\sigma_{h}=0.92 \mathrm{~cm}$, respectively. The neutral hydrogen gas species densities are fixed (uniform profiles) with $n_{\mathrm{H}_{2}}=8 \times 10^{19} \mathrm{~m}^{-3}, n_{\mathrm{H}}=2 \times 10^{19} \mathrm{~m}^{-3}$ together with temperatures of $T_{\mathrm{H}_{2}}=0.1 \mathrm{eV}$ and $T_{\mathrm{H}}=0.3 \mathrm{eV}$. Negative ions are produced by the dissociative attachment of a vibrationally excited hydrogen molecule. We assume that $1.5 \%$ of $\mathrm{H}_{2}$ molecules are in a vibrational state $\nu \geq 4$. Lastly, the magnetic field is uniform and parallel to the z-axis with a strength ranging from 100 to $400 \mathrm{G}$. $V_{e p}$ is typically negative to increase the residence time of an electron along a given magnetic field line.

the algorithm would be numerically unstable. In this work, the numerical resolution for the simulations ranges between $256^{2}$ and $1024^{2}$ grid nodes which corresponds to plasma densities $\sim 10^{14}$ and $10^{15} \mathrm{~m}^{-3}$, respectively. Plasma densities on axis in the RAID experiments or in the ITER prototype ion sources are typically around $\sim 10^{18} \mathrm{~m}^{-3}$ and hence about 3 orders of magnitude larger than in the simulations (or similarly an electron Debye length 30 times smaller). We are interested in studying particle dynamics in quiescent plasmas and as a consequence the plasma behaviour in the quasi-neutral volume will be similar regardless of the plasma density assuming that the particle collision mean-free-paths (mfp) stay constant. The principal difference is the length of the plasma sheath but its influence remains limited as long as its dynamics are preserved and the quasi-neutral volume largely exceeds the area occupied by the sheath. In 
this work, the sheath is collisionless and non-magnetised in both the model and the experiments for a magnetic field strength of up to $\sim 100 \mathrm{G}$. For larger magnetic fields, the question of the magnetisation of the electrons in the simulated sheath is an open problem (because we model lower plasma densities). Note that it is considered essential not to strongly magnetise the negative ions in order to facilitate their sideways extraction from the plasma through a slit aperture on the linear device cylinder wall and also due to the beam transport properties in the accelerator which will be deteriorated in case of magnetisation. The mfp for the collisions between charged particles and neutrals is independent of the simulated plasma density but not for the case of Coulomb interactions between electrons and ions. One hence needs to multiply the Rutherford scattering cross-sections by a scaling factor to correctly calculate the particle mfp, which is simply the ratio of the modelled plasma density divided by the target one. Another simpler and equivalent approach is to replace the vacuum permittivity constant $\varepsilon_{0}$ instead by $\varepsilon^{*}$ (i.e., a scaling by a factor $\alpha=\varepsilon^{*} / \varepsilon_{0}$ ) in Poisson's equation (in this case the plasma density in the model is equal to the experimental value one wishes to simulate),

$$
-\nabla^{2} \phi=\rho / \varepsilon^{*}
$$

where $\varepsilon^{*}$ is the permittivity in the model which only affects the length of the plasma sheath (artificially increased by $\sqrt{\alpha}$ ) in a quiescent plasma. Hereafter, we chose the latter approach. The inclusion of the electronelectron scattering process is left for future work. Note that it does not induce any transport across the magnetic field lines even in the presence of a density gradient. ${ }^{47,48}$

Figure 1 shows the simulation domain in the model. The linear device has a length $L_{z}=1 \mathrm{~m}$ and a radius $R=5 \mathrm{~cm}$. The numerical mesh is $2 \mathrm{D}$ Cartesian in the $(\mathrm{Ox})$ and $(\mathrm{Oy})$ directions while particle losses along the third dimension - $(\mathrm{Oz})$ - are calculated semianalytically as stated above. The area in light gray corresponds to the plasma location and the one in dark grey to the power absorption region using the profile defined by eq. (3). The magnetic field lines are straight, in the z-direction, and are terminated on the end-plates which are biased by a voltage $V_{e p}$. The magnetic field strength in the calculations analysed in this work ranges from 100 to $400 \mathrm{G}$ resulting in a situation where ions are either not or somewhat magnetised. Electrons are in all cases strongly magnetized with, for instance, a Hall parameter $h_{e}=\Omega_{c e} / \nu_{e} \sim 40$ for $\left|B_{z}\right|=100 \mathrm{G}$ where $\Omega_{c e}=e B_{z} / m_{e}$ is the electron cyclotron frequency and $\nu_{e}$ the total collision counterpart (which includes elastic, inelastic and ionisation). Electrons are hence well confined by the magnetic field and their residence time can be further increased by biasing negatively the end-plate potential $V_{e p}$. Ions are magnetised for $\left|B_{z}\right|=400 \mathrm{G}$ with a corresponding Hall parameter $h_{i} \sim 5$.
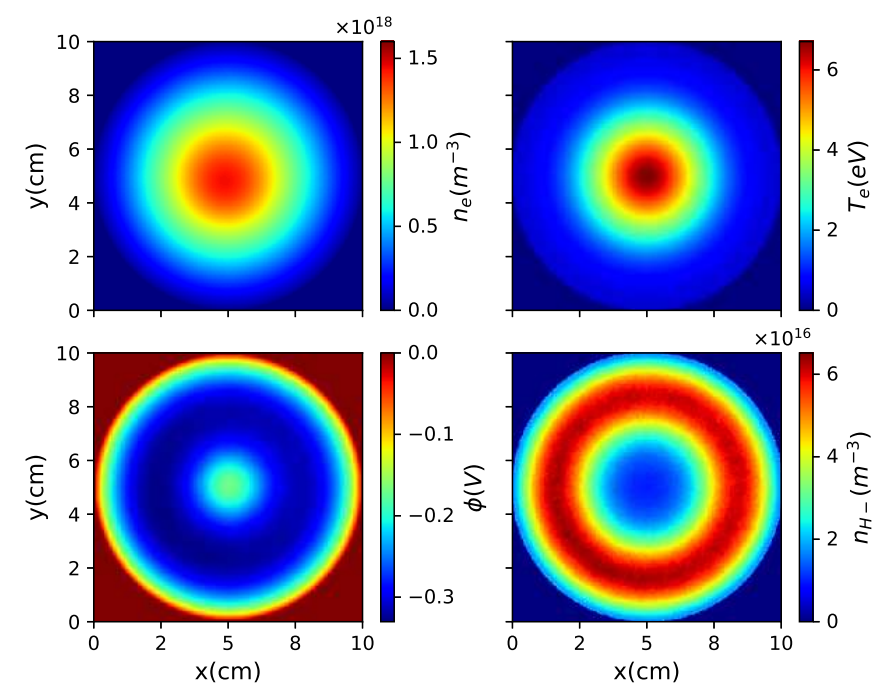

FIG. 2. 2D electron density, temperature, plasma potential and negative ion density profiles for the conditions of fig. 1 . $\left|B_{z}\right|=100 \mathrm{G}$ and the end-plates are floating with $V_{e p} \simeq-14 \mathrm{~V}$.

\section{INCIDENCE OF THE END-PLATE POTENTIAL ON THE PLASMA PROPERTIES}

In this section, we analyse the impact on the plasma properties of biasing negatively the metallic end-plates. We start by describing in detail a configuration where the end-plates are floating before performing a parameter scan. Note that we considered smaller dimensions than in the RAID experiment as it allows us to implement a higher numerical resolution.

\section{A. Floating end-plates}

Figure 2 shows the electron density, temperature, plasma potential and negative ion density $2 \mathrm{D}$ profiles for a plasma column with floating end-plates. The side wall of the cylinder is grounded. The numerical resolution of the calculation is $256^{2}$ grid nodes, 40 particles-per-cell (ppc), an average plasma density $\left\langle n_{p}\right\rangle \simeq 5.5 \times 10^{17} \mathrm{~m}^{-3}$, an absorbed power of $2.4 \mathrm{~kW}$, a standard deviation for the power density profile of $\sigma_{h}=0.92 \mathrm{~cm}$, a heating frequency $\nu_{h}=10^{7} \mathrm{~s}^{-1}$, a magnetic field strength of $100 \mathrm{G}$ and a scaling factor $\alpha=3200$ (corresponding to an electron Debye length to grid size ratio $\Delta x_{i} / \lambda_{D e} \simeq 0.5$ ), respectively. The background gas specie is hydrogen with a density of $n_{\mathrm{H} 2}=8 \times 10^{19} \mathrm{~m}^{-3}$, temperature $T_{\mathrm{H} 2}=0.1 \mathrm{eV}$ for the molecules, $n_{\mathrm{H}}=2 \times 10^{19} \mathrm{~m}^{-3}$ and a temperature $T_{\mathrm{H}}=0.3 \mathrm{eV}$ for the atoms. These temperatures are estimates based on experimental measurements and PIC-MCC calculations of fusion-type RF powered tandem type magnetised negative ion sources. ${ }^{18}$ Hydrogen atoms were found to have typically a larger temperature than the molecules due (1) to the Franck-Condon mech- 


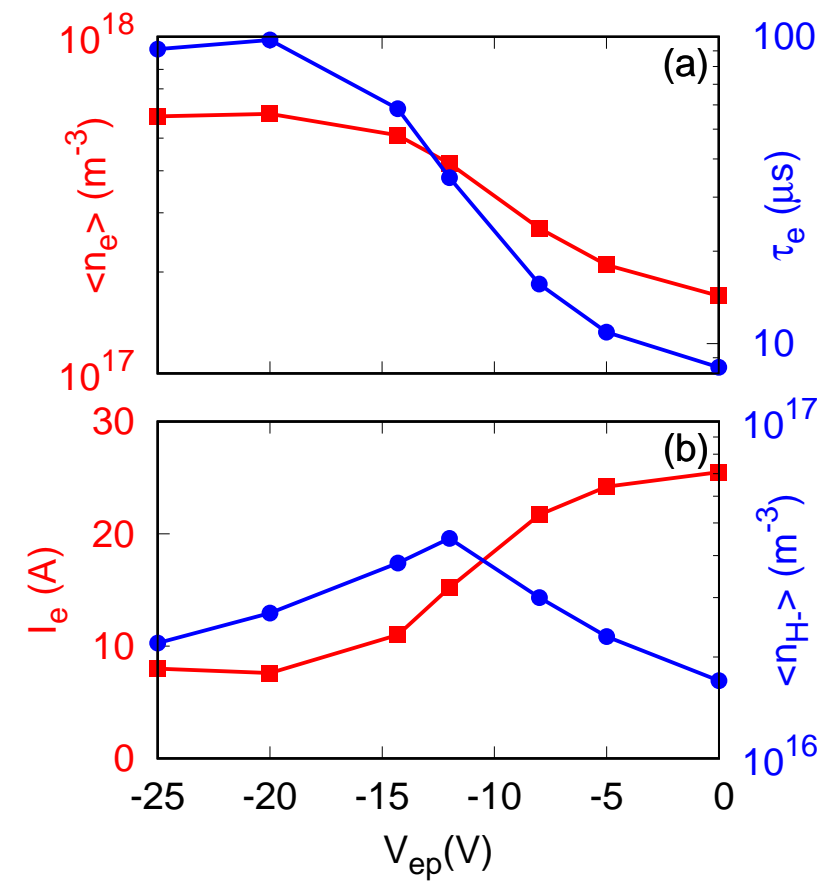

FIG. 3. (a) Electron residence time $\tau_{e}$ (blue solid line) inside the plasma of the linear device, volume averaged electron density $n_{e}$ (in red) and (b), volume averaged negative ion density $n_{H-}$ (in blue) together with the electron current impacting the cylinder walls (including the end-plates) versus the endplate bias voltage $V_{e p}$.

anism which produces fast atoms as a byproduct of a dissociative collision between $\mathrm{H}_{2}$ and electrons ${ }^{33}$ and (2) in low background gas pressure working conditions as a result of the recombination of fast ions impacting surfaces when the plasma potential is large (typically $\sim 40 \mathrm{~V}$ inside the discharge of ITER-prototype plasma sources for instance). As stated above, the proportion of hydrogen molecules in a vibrational state $\nu \geq 4\left(\mathrm{H}_{2}^{*}\right)$ is assumed to be $1.5 \%$ of the background density. Negative ions are produced inside the ion source volume by the dissociative attachment process of $\mathrm{H}_{2}^{*}$ (reaction \#17 of table I). Lastly, the time step is $\Delta t \simeq 0.2 \mathrm{~ns}$ and convergence is reached in about $100 \mu \mathrm{s}$. The floating potential at steady state is $V_{e p} \simeq-14 \mathrm{~V}$ and is calculated self-consistently from the charges accumulating on the end-plates,

$$
V_{e p}=C^{-1} \sum_{s} q_{s}\left(z_{e p}, T\right),
$$

where $z_{e p}$ is the physical location of the plates, $q_{s}\left(z_{e p}, T\right)$ the total charge collected on the surfaces during a time interval $T$ for each particle species $s$ and $C$ a numerical capacitance. The capacitance should be chosen large enough to modify the potential value only after an integration over a significant number of time steps (this will reduce fluctuations). The voltage in eq. (6) self-adjusts until steady-state conditions are reached. We assumed a value $C=10^{-6} \mathrm{~F}$, corresponding for instance to a potential increase of $\Delta V_{e p}=1 \mathrm{~V}$ for $I=1 \mathrm{~A}$ of net current

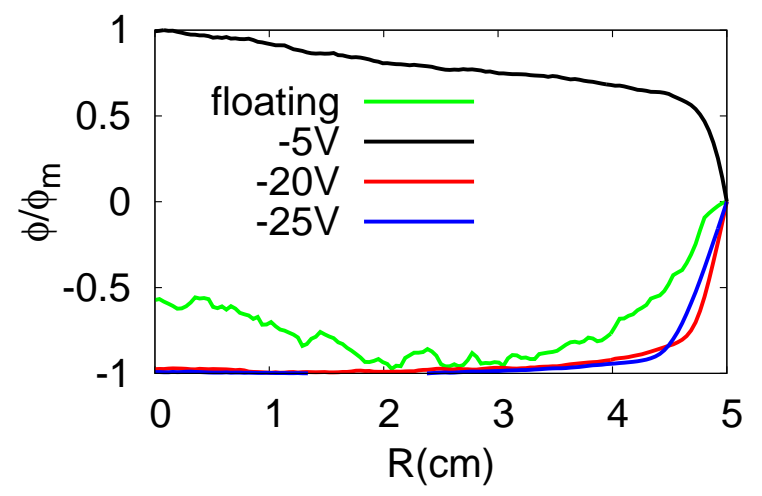

FIG. 4. Normalized radial plasma potential profiles versus the end-plate bias voltage for the conditions of fig. 1 . The floating potential corresponds to a voltage of $\simeq-14 \mathrm{~V}$. The normalisation factor $\phi_{m}$ is equal to $4.1 \mathrm{~V}$ for a bias voltage of $-5 \mathrm{~V},-0.4 \mathrm{~V}$ for a floating end-plate, $-3.4 \mathrm{~V}$ and lastly $-7.7 \mathrm{~V}$ for end-plate potentials of $-20 \mathrm{~V}$ and $-25 \mathrm{~V}$, respectively. The potential is somewhat uniform inside the quasi-neutral plasma region in the last two cases, with variations below $0.1 \mathrm{~V}$ in the calculation.

impacting the surface over $\sim 5000$ time steps $\Delta t$ (which is equivalent to about $1 \mu \mathrm{s}$ ) with

$$
\Delta V_{e p}=\frac{I \Delta t}{C} .
$$

Note that transient effects occurring on time scales shorter than the capacitor response will be smoothed out (which is not an issue in our case as we are investigating steady-state situations ou slower plasma dynamics).

The amplitude of the plasma potential in fig. 2 is found to be close to $0 \mathrm{~V}$; its profile has a u-shape radially with a difference between the maximum at $r=\sqrt{x^{2}+y^{2}}=0$ and the minimum around $2.8 \mathrm{~cm}$ of $\Delta \phi \simeq 0.15 \mathrm{eV}$. This may confine electrostatically some positive ions (these particles will be lost on the end-plates instead). The plasma density is maximum on the linear device axis with $n_{p} \simeq 1.5 \times 10^{18} \mathrm{~m}^{-3}$ while the electron temperature $T_{e}$ is $6.5 \mathrm{eV}$ and decreases to about $1 \mathrm{eV}$ at the location where the negative ion density profile peaks. The latter has a donut-like shape with a density of $n_{\mathrm{H}^{-}} \simeq 10^{16} \mathrm{~m}^{-3}$ on axis and a maximum 6 times larger at a radius $r=$ $3.4 \mathrm{~cm}$. This is the consequence of the cross-section of reaction \#17 in table $\mathrm{I}$, which is largest for $T_{e} \simeq 0.3 \mathrm{eV}$ $\left(\sigma \simeq 10^{-15} \mathrm{~cm}^{2}\right)$, is halved for an electron temperature of $1 \mathrm{eV}$ and is 4 orders of magnitude lower for $5 \mathrm{eV}{ }^{33}$

\section{B. Scan versus the end-plate voltage}

The simulations performed in this subsection are for a magnetic field strength of 100G. Electrons are strongly magnetized with a Hall parameter $h_{e} \sim 40$ (corresponding to a gyroradius $\rho_{e}=v_{t h} / \Omega_{e} \simeq 0.6 \mathrm{~mm}$ for $T_{e}=3$ $\mathrm{eV})$. Electrons are hence attached to a magnetic field line and slowly diffuse perpendicular to the latter through 


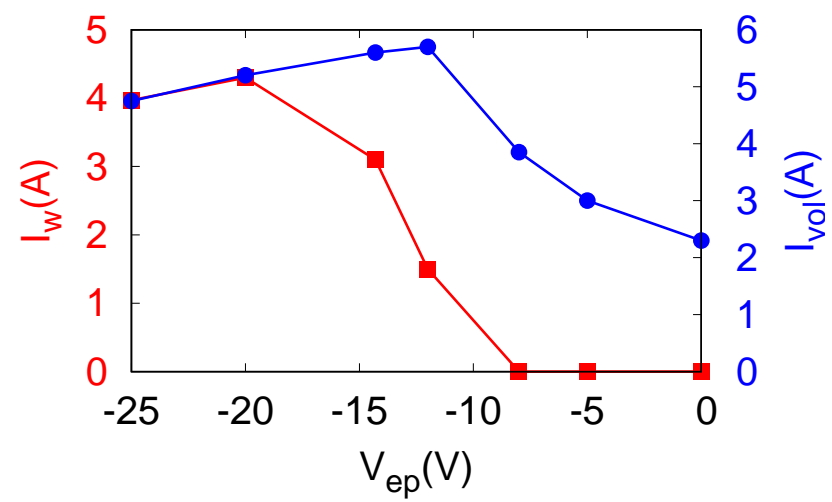

FIG. 5. Negative ion current either impacting the discharge walls $\left(I_{w}\right)$ or lost inside the plasma volume $\left(I_{\mathrm{vol}}\right)$ versus the end-plate bias voltage $V_{e p}$.

collisions against ions (Coulomb) and neutrals (the ionisation fraction being less than $1 \%$ in the model). The magnetic field lines are short-circuited by the end-plates and furthermore electrons can gain energy axially, i.e., along $(\mathrm{Oz})$, and hence escape across the plasma sheath in that direction. One way to reduce losses and consequently to lengthen the residence time of the electrons is to bias negatively the end-plates such as to increase the height of the sheath potential drop. This is shown in fig. 3 where the average electron residence time $\tau_{e}$ is increased by a factor $\sim 10$ when the end-plate voltage varies from $V_{e p}=0 \mathrm{~V}$ to $-20 \mathrm{~V}$ while the average electron density increases from $\left\langle n_{p}\right\rangle \simeq 1.7 \times 10^{17} \mathrm{~m}^{-3}$ to $6 \times 10^{17} \mathrm{~m}^{-3}(2.4$ $\mathrm{kW}$ absorbed power), respectively. The simulation parameters are provided in fig. 1 together with a numerical resolution of $256^{2}$ grid nodes, $40 \mathrm{ppc}$, a heating frequency $\nu_{h}=10^{7} \mathrm{~s}^{-1}$ and a scaling factor $\alpha=3200 . \tau_{e}$ is calculated by integrating the electron continuity equation over the plasma volume (assuming steady-state conditions), that is,

$$
I_{w}=\int_{S} e n_{e} u_{e} d S=\int_{V} e n_{e} \nu_{i} d V
$$

where $I_{w}$ is the electron current impacting the device walls, $S$ is the corresponding wall surface, $u_{e}$ the electron velocity at the wall, $V$ the plasma volume and $\nu_{i}$ the net electron production frequency inside the plasma (which accounts for the balance between ionisation and losses). Further assuming that $\tau_{e}=\nu_{i}^{-1}$ is constant over the entire volume, one get,

$$
\tau_{e}=\frac{e \pi R^{2} L_{z}\left\langle n_{e}\right\rangle}{I_{w}}
$$

with,

$$
\left\langle n_{e}\right\rangle=\left(\pi R^{2} L_{z}\right)^{-1} \int_{V} n_{e} d V
$$

Forcing electrons to diffuse across the magnetic field lines and to be lost on the linear device cylinder walls instead

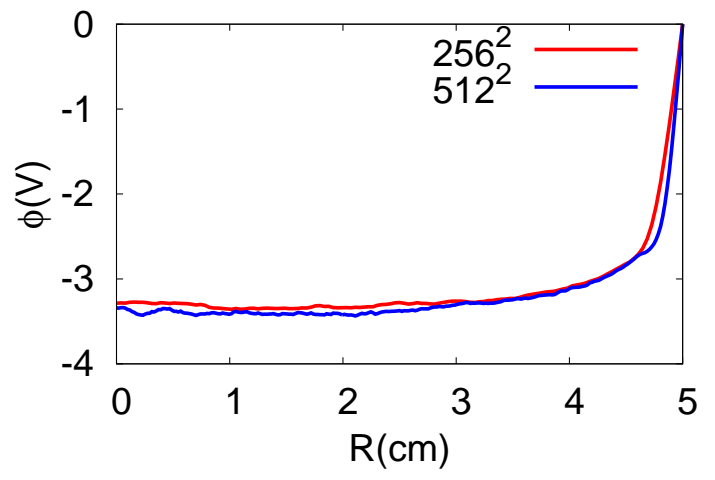

FIG. 6. Radial plasma potential profile for an end-plate bias voltage $V_{e p}=-20 \mathrm{~V}$ and a scaling factor either of $\alpha=3200$ (corresponding to a $256^{2}$ grid nodes resolution) or $800\left(512^{2}\right.$ nodes), respectively.

of on the end-plates has an impact on the plasma potential profile as shown in fig. 4. The height of the plasma potential decreases when the end-plate bias voltage is increased (in absolute value). The potential profile eventually becomes a well for the ions (it has a u-shape); the transition occuring when the end-plates are floating in the model. Positive ions are then lost on the end-plates because the depth of the potential well largely exceeds the ion temperature. The volume averaged electron density as well as the electron current impacting the device walls are saturating when the end-plates' bias voltage is greater than a couple of times the electron temperature (in electron-Volts) as shown in fig. 3(a) and (b).

The electron residence time is correlated with the probability of generating a negative ion via the dissociative attachment of the hydrogen molecule by electron impacts. The longer the electrons stay in the plasma, the higher the negative ion production yield. Figure 5 shows the negative ion current lost in volume by collisions (reaction \#16 of table I, \#10, 11, 15 and 16 of table II, respectively) and on the walls of the device (including the endplates). The production rate is the sum of the two currents. The total negative ion current jumps from $\sim 2 \mathrm{~A}$ for end-plates at ground potential up to $\sim 10 \mathrm{~A}$ for $-20 \mathrm{~V}$, respectively, which is the maximum. For comparison the discharge current is $\sim 12 \mathrm{~A}$ (summed over the positive ion currents) and the electron current lost on the walls is $\sim 7.5 \mathrm{~A}$ for the same bias $(-20 \mathrm{~V})$, indicating that the negative ion yield may be substantial. Note that this does not translate into a large negative ion density because a large fraction of these ions is destroyed through collisions with electrons and neutrals. As a consequence, negative ion losses inside the plasma volume amount to more than $50 \%$ of the production rate regardless of the value of the end-plate bias voltage as shown in fig. 5 . This is a disadvantage of volume production of negative ions compared with surface production on the extractor electrodes. The ion to electron volume averaged density ratio $\left\langle n_{\mathrm{H}^{-}}\right\rangle /\left\langle n_{e}\right\rangle$ is less than $10 \%$ in the calculations (see fig. 3). Furthermore, the plasma potential confines negative charges for 
configurations where the end-plate voltage lies between ground potential and floating conditions (fig. 4) but beyond that point, the potential accelerates negative ions radially. The latter hence increasingly convey the negative charges toward the cylinder wall, which is correlated in turn with a decrease of both the losses in volume and the negative ion density (see fig. 3). Note that there aren't any negative ion losses on the end-plates.

The model is simulating plasma densities of $\sim 5 \times$ $10^{17} \mathrm{~m}^{-3}$ on average with a plasma sheath larger than real due to the implementation of a scaling factor $\alpha=$ $\varepsilon^{*} / \varepsilon_{0}$ in order to artificially increase the size of the electron Debye length and hence reduce the number of grid nodes. Figure 6 shows the plasma potential profile versus a numerical resolution of $256^{2}$ and $512^{2}$ nodes corresponding to a factor $\alpha=800$ and 3200, respectively. In both cases, the potential well is accurately reproduced with a similar width (defined as the cylinder radius minus the plasma sheath length) and depth. Lastly, an a-posteriori verification of the EEDF shows that the numerical technique simulating the RF power absorption by the macro-electrons in the model generates a local Maxwellian distribution. A detailed inclusion of the RF-plasma coupling dynamics might result in a nonMaxwellian EEDF which will affect the inelastic collision rates, impacting in turn the dissociative attachment and hence the negative ion density but the conclusions derived in this work from the parameter scans will remain identical. Figure 7 shows the EEDF in both the region of power absorption and around the location where the negative ion density is maximum for the case of an end-plate bias voltage of $-8 \mathrm{~V}$.

\section{COMPARISON WITH EXPERIMENTS ON THE RAID LINEAR DEVICE}

Figure 8(a) plots the normalised electron density $n_{e} / n_{e m}$, temperature $T_{e} / T_{e m}$ and negative ion density $n_{\mathrm{H}^{-}} / n_{m}$ profiles obtained with the $2.5 \mathrm{D}$ PIC-MCC model for the conditions of the experiments on the RAID testbed with $n_{e m}=1.5 \times 10^{18} \mathrm{~m}^{-3}, T_{e m}=5.2 \mathrm{eV}$ and $n_{m}=1.7 \times 10^{16} \mathrm{~m}^{-3}$, respectively. The absorbed power is assumed to be $3.1 \mathrm{~kW}$ with a Gaussian profile and a standard deviation of $\sigma_{h}=1.5 \mathrm{~cm}$ (best fit in order to reproduce the experimental electron temperature profile). RAID has a $20 \mathrm{~cm}$ radius and a plasma column length $L_{z}=1.5 \mathrm{~m}$. The calculation of fig. 8 corresponds to a magnetic field strength of $200 \mathrm{G}$ and end-plates at ground potential. The simulation parameters are a mesh of $512^{2}$ nodes, $100 \mathrm{ppc}$, a heating frequency $\nu_{h}=10^{8} \mathrm{~s}^{-1}$ and a scaling factor $\alpha=3200$. The characteristics of the hydrogen gas are given in fig. 1. Furthermore, we fixed the proportion of $\mathrm{H}_{2}^{*}$ which are vibrationally excited $(\nu \geq 4)$ to $0.25 \%$ of the total density (which is an external parameter to the model) to approximately fit the peak value of the negative ion density observed in the experiments.

The electron density and temperature in the experi-

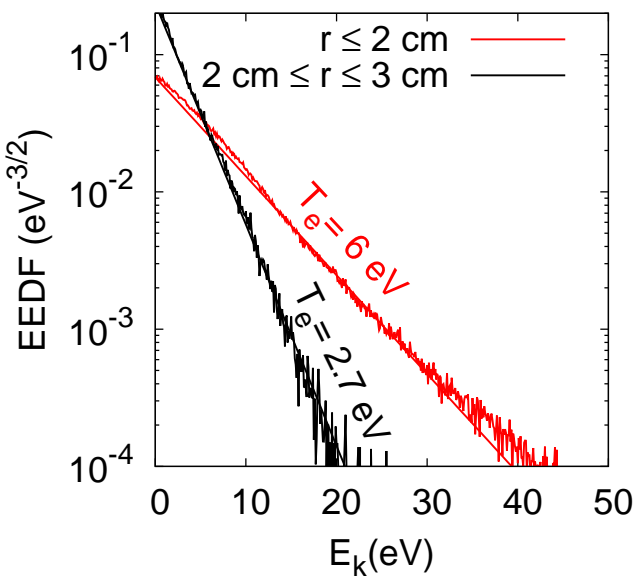

FIG. 7. Electron Energy Distribution Function (EEDF) profile in the power absorption region $(r \leq 2 \mathrm{~cm})$ and near the maximum in negative ion density $(2 \mathrm{~cm} \leq r \leq 3 \mathrm{~cm})$. The straight lines are Maxwellian fits. The magnetic field strength is $100 \mathrm{G}$ and the end-plate bias voltage $-8 \mathrm{~V}$, respectively.

ment are derived from axially movable Langmuir probe (LP) measurements calibrated with interferometry while the absolute negative ion density is obtained using Cavity Ring Down Spectroscopy (CRDS) ${ }^{49}$ combined with LP-assisted laser photodetachment. ${ }^{16}$ The experimental electron and negative ion density radial profiles together with the electron temperature (dashed-lines) are shown in fig. 8(a) for comparison with the estimates from the 2.5D PIC-MCC calculation (solid lines) while the plasma potential is plotted in fig. $8(\mathrm{~b}) . n_{e m}=2 \times 10^{18} \mathrm{~m}^{-3}$, $T_{e m}=4.3 \mathrm{eV}, n_{m}=1.1 \times 10^{16} \mathrm{~m}^{-3}$ and the error bar for the plasma potential is $\pm 1 \mathrm{~V}$ in the experiment. The model is hence capable of reproducing the general characteristics observed in the experiments, i.e., a similar amplitude and profile for the plasma parameters. In addition, the maximum plasma potential on axis is about $11.5 \mathrm{~V}$ in the calculation compared to $\sim 12 \mathrm{~V}$ for a radius $R<6 \mathrm{~cm}$ in RAID. The negative ion density profile peaks in both cases in the area where the electron temperature drops to $\sim 1 \mathrm{eV}$ and is significantly lower on the discharge axis. This indicates an annular profile similar to the one of fig. 2. Note that the ions are somewhat magnetized which results in the formation of rotating structures in the model in the area where the plasma pressure gradient is significant $(R<6 \mathrm{~cm})$. A similar behaviour has been observed in the experiments on RAID but in Argon gas and for a magnetic field strength of $800 \mathrm{G} .{ }^{50}$

\section{EFFECT OF THE ION MAGNETISATION ON THE PLASMA PROPERTIES}

In this section, we analyse the incidence on the plasma characteristics of magnetising the positive ions. One obtains quiescent plasma conditions in the linear device model when the ions remain unmagnetized as shown 

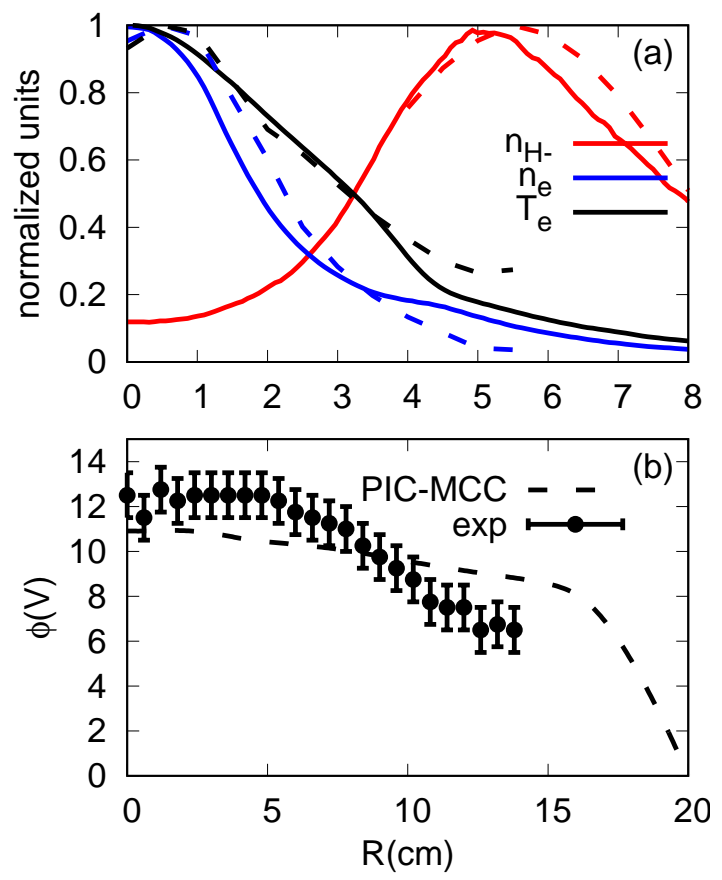

FIG. 8. Normalised radial profiles for the electron density $n_{e} / n_{e m}$, temperature $T_{e} / T_{e m}$ and negative ion density $n_{\mathrm{H}^{-}} / n_{m}$ in (a). Comparison between the RAID experiments (dashed lines) versus the 2.5D PIC-MCC calculation (solid lines). $n_{e m}=1.5 \times 10^{18} \mathrm{~m}^{-3}, T_{e m}=5.2 \mathrm{eV}, n_{m}=$ $1.7 \times 10^{16} \mathrm{~m}^{-3}$ in the simulations and $n_{e m}=2 \times 10^{18} \mathrm{~m}^{-3}$, $T_{\text {em }}=4.3 \mathrm{eV}$ and $n_{m}=1.1 \times 10^{16} \mathrm{~m}^{-3}$ for the experiments, respectively. The plasma potential is displayed in (b). The error bar is $\pm 1 \mathrm{~V}$. Lastly, the absorbed power profile in the model is Gaussian with a standard deviation of $1.5 \mathrm{~cm}$ and an integrated power of $3.1 \mathrm{~kW}$.

in fig. 2. Increasing the strength of the magnetic field lengthens the residence time of the electrons as their transverse mobility is lowered (the Hall parameter evolves from $h_{e} \sim 40$ to $\sim 160$ between $100 \mathrm{G}$ and 400G, respectively). This in turn results in higher plasma densities (for the same absorbed power) and narrower plasma profiles. This is shown by comparing fig. 2 and fig. 9 which corresponds to identical simulations except for the magnetic field strength, which is $400 \mathrm{G}$ in the latter case. Note that we have performed a convergence test for this configuration and did not find any noticeable differences when increasing the number of particles per cell to 400 instead of 40 or changing the number of grid nodes from $256^{2}$ to $512^{2}$, respectively. Similar conclusions were drawn when studying the effect of the artificial heating frequency $\nu_{h}$ (in a configuration with a simplified physical-chemistry) where we varied the fraction of macro-electrons in the heating region which absorbs the external power from $\nu_{h} \Delta t=0.1 \%$ to $1 \%$ without loss of generality.

A transition in plasma behaviour occurs when the ions become somewhat magnetized. ${ }^{51}$ In fig. 9 , one observes the formation of large scale structures and the plasma is
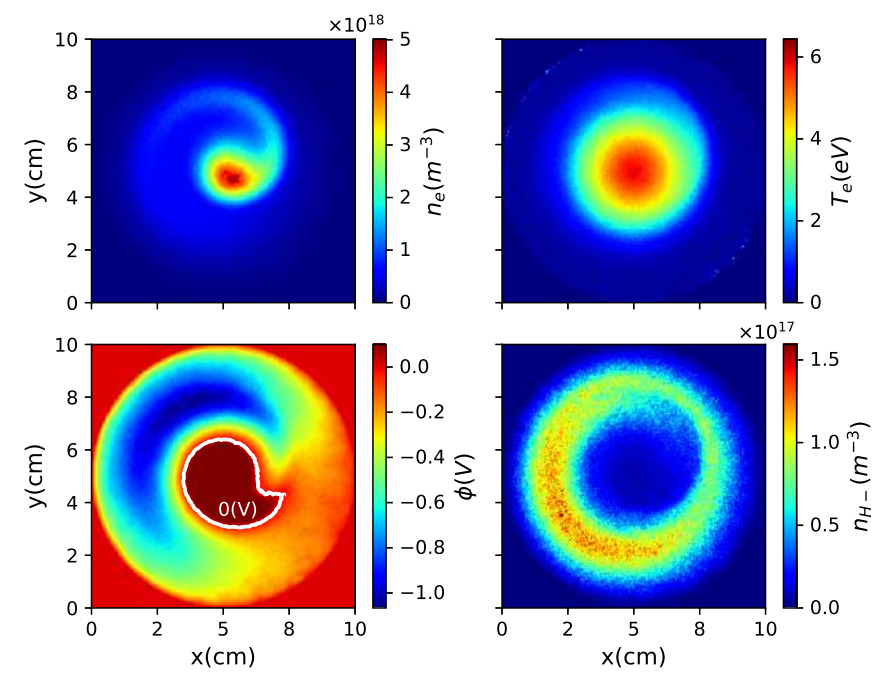

FIG. 9. 2D electron density, temperature, negative ion density and plasma potential profiles for the conditions of fig. 1 . $\left|B_{z}\right|=400 \mathrm{G}$ and the end-plates are floating with $V_{e p} \simeq-14 \mathrm{~V}$. The volume occupied by the plasma sheath is slightly below $10 \%$ of the simulation domain due to the use of a scaling factor $\alpha=3200$.

rotating clockwise with a period of $\sim 75 \mu \mathrm{s}$ (13 kHz frequency). The entire plasma volume (except the sheath), including the rotating arm is quasi-neutral and hence the Debye length is not a critical parameter, justifying the use of a scaling factor for the vacuum permittivity in the model without any detrimental effects on the plasma dynamics. The diamagnetic drift velocity,

$$
v_{D \beta}=-\frac{\nabla P_{\beta} \times \mathbf{B}}{q_{\beta} n_{\beta} B^{2}},
$$

leads to a counterclockwise rotation for the electrons $(\beta$ corresponds to any charged particle species and $q_{\beta}$ its respective charge). The radial electric field $E_{r}$ is positive inside the bulk where the rotating arm is located while the pressure gradient is negative everywhere. The structure hence rotates azimuthally in the $\mathbf{E} \times \mathbf{B}$ direction (taking $B_{z}>0$ ). The arm rotation appears to be approximately rigid, i.e., with the same frequency regardless of its radial location. The corresponding velocity hence varies linearly radially and is about $2 \mathrm{~km} / \mathrm{s}$ for instance at mid-radius which is the same order of magnitude as the average ion velocity; the latter being defined as,

$$
u_{i}=\sum_{\beta}\left(n_{\beta} u_{\beta}\right) / \sum_{\beta} n_{\beta},
$$

where $u_{\beta}$ is the $\beta$ ion specie average velocity and $n_{\beta}$ the corresponding density. The ions also rotate clockwise with a velocity profile comparable to the $\mathbf{E} \times \mathbf{B}$ drift. Their respective root-mean-square (RMS) velocities, $\sqrt{\left\langle u_{i}^{2}\right\rangle}$ and $\sqrt{\left\langle v_{E}^{2}\right\rangle}$ calculated inside a disk of radius $r \leq 4 \mathrm{~cm}$ and a center aligned with the discharge axis (in order to exclude the plasma sheath) are about $1 \mathrm{~km} / \mathrm{s}$ in 


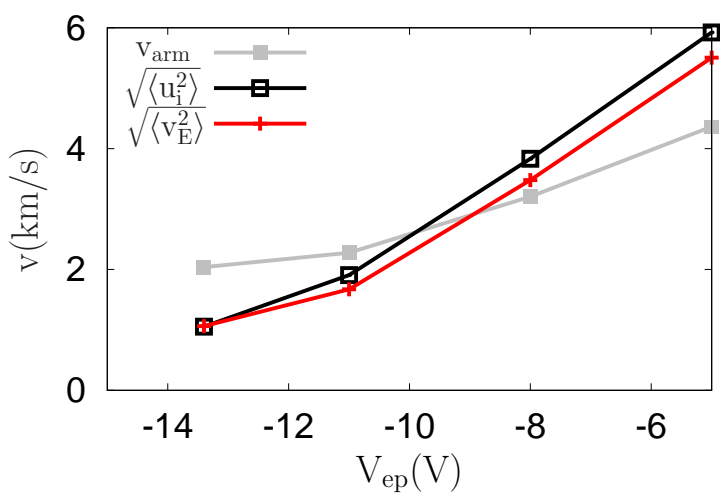

FIG. 10. Rotation velocity of the large scale structures measured at mid-radius $r=2.5 \mathrm{~cm}$ (grey), root-mean-square (RMS) average ion velocity (black) and $\mathbf{E} \times \mathbf{B}$ drift velocity (red) versus the linear device end-plate bias voltage. The RMS velocities are calculated inside a radius $r \leq 4 \mathrm{~cm}$.

both cases where

$$
\mathbf{v}_{E}=\frac{\mathbf{E} \times \mathbf{B}}{B^{2}} .
$$

For comparison, the RMS proton sound speed $\sqrt{\left\langle u_{B}^{2}\right\rangle} \simeq$ $13 \mathrm{~km} / \mathrm{s}\left(u_{B}=\sqrt{e T_{e} / m_{i}}\right)$ and the RMS average electron velocity $\sqrt{\left\langle u_{e}^{2}\right\rangle} \sim 18 \mathrm{~km} / \mathrm{s}$. Figure 10 plots the rotating arm velocity together with the RMS ion and $\mathbf{E} \times \mathbf{B}$ drift velocities versus the polarisation of the end-plate bias plates. One observes that $\sqrt{\left\langle u_{i}^{2}\right\rangle}$ and $\sqrt{\left\langle v_{E}^{2}\right\rangle}$ are similar and decrease for larger bias voltages (in absolute value) which is caused by a lower plasma potential amplitude as shown in fig. 4 . The arm velocity is also decreasing but at a smaller pace with respect to the ion velocity. The detailed assessment of the physics leading to the formation of these large scale rotating structures and the associated modified plasma transport properties (notably the question of anomalous current) is left to future work. Note that we observe similar behaviours in electro-positive gases, which have also been detected experimentally ${ }^{52,53}$, and hence the negative ions do not seem to be seeding these instabilities although they likely affect the latter (this is currently an open question). The negative ions have a ring-like density profile (fig. 9) which is also rotating but distorted by the varying electric field strength along their path. The electronegativity is $n_{\mathrm{H}^{-}} / n_{e} \sim 0.5$ near the peak indicating that the negative ions represent a significant fraction of the negative charges in that area.

\section{EXTRACTION OF NEGATIVE IONS}

Figure 5 shows that negative ions are trapped inside the plasma volume and destroyed by collisions with charged particles and neutrals when the plasma potential amplitude is above the linear device cylinder wall voltage (which is at ground potential in the calculation). Biasing the end-plates negatively acts as a control knob chang-
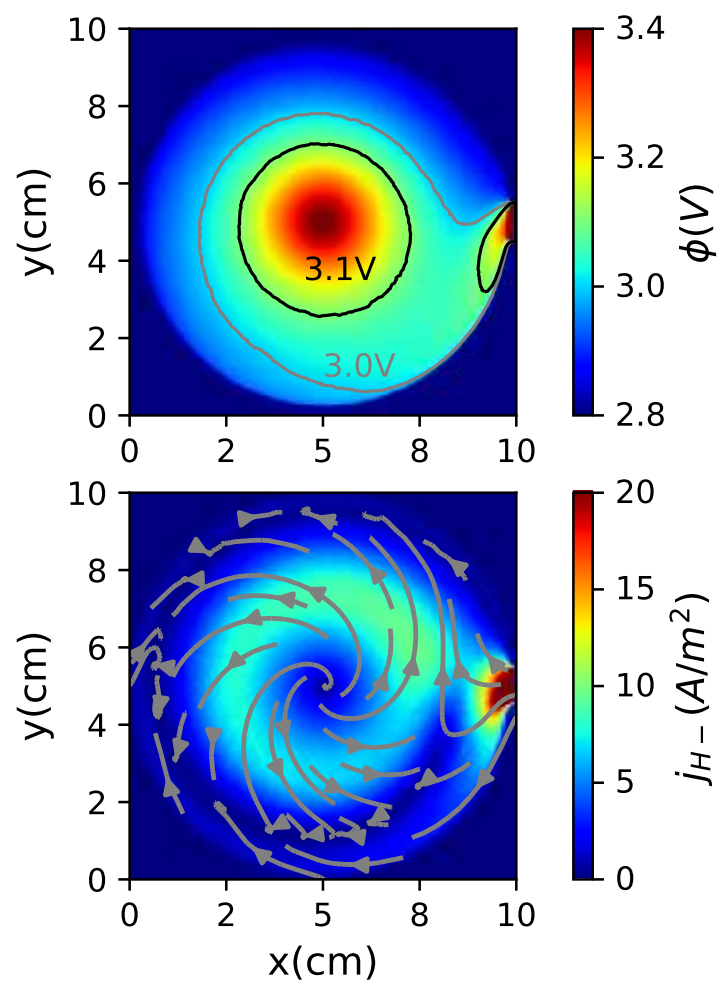

FIG. 11. 2D plasma potential and negative ion current density profiles inside the plasma volume of the linear device. The current streamlines are shown in grey (lower plot). The simulation corresponds to a cylinder radius of $5 \mathrm{~cm}$, an endplate potential of $-10 \mathrm{~V}$, a slit aperture width of $1 \mathrm{~cm}$ and a bias of $5 \mathrm{~V}$, respectively. The numerical resolution is $1024^{2}$ grid nodes. $j_{\mathrm{H}^{-}} \simeq 35 \mathrm{~A} / \mathrm{m}^{2}$ on the slit aperture.

ing the shape of the radial plasma potential profile such that negative ions may be directly extracted. The latter approach is not interesting for an ion source as the ion radial flux has a cylindrical symmetry. In a Neutral Beam Injector, one would need to extract the ions through slit or cylindrical apertures embedded on the cylinder surface and connected to an electrostatic accelerator in order to accelerate the particles to high energies ( $1 \mathrm{MeV}$ in the case of ITER for instance). Note that a similar extraction scheme for negative ions produced in a linear device has been studied experimentally using $\mathrm{SF}_{6}$ gas. ${ }^{54}$ The numerical resolution of the PIC algorithm cannot implement the details of the aperture geometry. We therefore chose to model instead the impact on the negative ion dynamics of a small surface on the cylinder wall which is biased positively with respect to the plasma potential. This mimics the penetration of the accelerator extraction voltage inside the plasma, in the vicinity of the meniscus. The biased surface has a width of $1 \mathrm{~cm}$ in the model and a voltage of $5 \mathrm{~V}$. The numerical resolution is $1024^{2}$ grid nodes, $40 \mathrm{ppc},-10 \mathrm{~V}$ end-plate bias potential, a heating frequency $\nu_{h}=10^{7} \mathrm{~s}^{-1}$ and a scaling factor $\alpha=50$. The remaining parameters are identical to those of fig. 1 . The maximum value of the electron Debye length in the cal- 
culation is about $100 \mu \mathrm{m}$ and the time step $\Delta t \simeq 50 \mathrm{ps,}$ respectively. Figure 11 plots the $2 \mathrm{D}$ plasma potential profile inside the linear device together with the negative ion current density. The plasma potential increases sharply near the biased slit as expected (RHS of the plot) but there is also a minimum around mid-radius with respect to the amplitude on axis $(\Delta \phi \simeq 0.4 \mathrm{~V})$. This traps a sub-population of negative ions in that area which therefore cannot be extracted. The average negative ion current density on the slit aperture is $j_{\mathrm{H}^{-}} \simeq 35 \mathrm{~A} / \mathrm{m}^{2}$ (i.e., $7 \%$ of the negative ion current produced in the plasma volume), which is an order of magnitude lower than the ITER NBI requirement for instance. $j_{\mathrm{H}^{-}}$is correlated with a maximum negative ion density of $4 \times 10^{16} \mathrm{~m}^{-3}$ about $1.5 \mathrm{~cm}$ from the cylinder's surface. The possibility to extract a larger current remains an open question as the negative ions are produced inside the plasma volume as opposed to the direct vicinity of the apertures where the ions are scattered off a cesiated surface after the impact of a neutral or a positive ion. The negative ion density profile peaks in the region where the electron temperature falls below $1 \mathrm{eV}$ in the model. The location of the peak may hence be controlled and shifted toward the cylinder walls by modifying the extent of the power absorption region.

\section{CONCLUSION}

We studied the feasibility of producing and extracting a hydrogen negative ion current generated inside the plasma volume of a linear device (through physicalchemistry processes between charged particles and the background gas) with a magnitude relevant to the requirements of magnetic fusion Neutral Beam Injectors (NBI). Linear devices are interesting configurations as the electrons are strongly magnetised with field lines aligned with the discharge axis. The large aspect ratio of the discharge (defined as the axial length over the cylinder radius) implies that electrons will oscillate between the end-plates located at the extremities of the cylinder and diffuse slowly across the magnetic field. The residence time of the electrons inside the ion source has an incidence on the production yield of the negative ions. Negative ions are produced inside the plasma in a two step process consisting of (i) an inner volume with an electron temperature between 5 and $10 \mathrm{eV}$ where the hydrogen background gas is excited vibrationally and (ii), an outer volume, with a temperature below $1 \mathrm{eV}$ where the negative ions are generated as a byproduct of the dissociative attachment of a hydrogen molecule due to a collision with an electron. We showed that biasing negatively the end-plates increases the electron residence time by an order of magnitude and as a consequence the negative ion density may be increased by a factor $\sim 3$ for a given absorbed RF power. Biasing the end-plates negatively causes a reduction of the amplitude of the plasma potential which is a limiting factor for the negative ion density as the potential profile switches to a well when the end-plate voltage is such that one collects a higher ratio of positive ions compared to electrons. In that case, the plasma potential extracts radially the negative ions. One observes that the negative ion density has a ring-like shape with a maximum at the location where the electron temperature falls below $1 \mathrm{eV}$. This is consistent with experimental measurements on the RAID linear device. The position of the maximum may be moved by adjusting the radius of the power absorption region in the model. We simulated the extraction of negative ions by biasing positively $(+5 \mathrm{~V})$ a surface on the cylinder wall with a width of $1 \mathrm{~cm}$. The extracted ions amounted to about $7 \%$ of the total current produced in the source volume but this remained about an order of magnitude lower that the value fulfilling the requirement for the ITER NBI for instance (which is $\sim 250 \mathrm{~A} / \mathrm{m}^{2}$ ). To which extent increasing the helicon power is correlated also with a larger negative ion density in the experiments (before reaching any saturation mechanisms induced among others by neutral depletion in low pressure conditions, physical-chemistry, etc.) remains an open question. Lastly, large scale rotating structures appear when the ions become somewhat magnetised. These instabilities rotate at a frequency between 13 and $29 \mathrm{kHz}$ in the calculation. The latter increases in correlation with a larger plasma potential amplitude suggesting a relationship with the $\mathbf{E} \times \mathbf{B}$ drift and hence the ion velocity. The detailed understanding of the physical mechanisms seeding these instabilities is left to future work.

\section{ACKNOWLEDGMENTS}

This work has been carried out within the framework of the EUROfusion Consortium and has received funding from the Euratom research and training programme 2014-2018 and 2019-2020 under grant agreement No 633053. The views and opinions expressed herein do not necessarily reflect those of the European Commission. This work was supported in part also by the Swiss National Science Foundation, Commissariat à l'Énergie Atomique (CEA) and the French Fédération de Recherche sur la Fusion Magnétique (FR-FCM).

\section{AVAILABILITY OF DATA}

The data that support the findings of this study are available from the corresponding author upon reasonable request.

\footnotetext{
${ }^{1}$ M. Bacal and M. Wada, Applied Physics Reviews 2, 021305 (2015).

${ }^{2}$ M. Bacal and M. Wada, Plasma Sources Sci. Technol. 29, 033001 (2020).

${ }^{3}$ D. Wünderlich, R. Riedl, I. Mario, A. Mimo, U. Fantz, B. Heinemann, and W. Kraus, Review of Scientific Instruments 90, 113304 (2019).
} 
${ }^{4}$ A. Ueno, H. Oguri, K. Ikegami, Y. Namekawa, and K. Ohkoshi, Review of Scientific Instruments 81, 02A720 (2010).

${ }^{5}$ D. P. Moehs, J. Peters, and J. Sherman, IEEE Transactions on Plasma Science 33, 1786 (2005).

${ }^{6}$ J. Lettry, D. Aguglia, P. Andersson, S. Bertolo, A. Butterworth, Y. Coutron, A. Dallocchio, E. Chaudet, J. Gil-Flores, R. Guida, J. Hansen, A. Hatayama, I. Koszar, E. Mahner, C. Mastrostefano, S. Mathot, S. Mattei, Ø. Midttun, P. Moyret, D. Nisbet, K. Nishida, M. O'Neil, M. Ohta, M. Paoluzzi, C. Pasquino, H. Pereira, J. Rochez, J. Sanchez Alvarez, J. Sanchez Arias, R. Scrivens, T. Shibata, D. Steyaert, N. Thaus, and T. Yamamoto, Review of Scientific Instruments 85, 02B122 (2014).

${ }^{7}$ R. F. Welton, A. V. Aleksandrov, V. G. Dudnikov, B. X. Han, Y. Kang, S. N. Murray, T. R. Pennisi, C. Piller, M. Santana, and M. P. Stockli, Review of Scientific Instruments 87, 02B146 (2016).

${ }^{8}$ M. Yoneda, Y. Shibata, A. Tanaka, T. Uehiro, M. Morita, M. Uchida, T. Kobayashi, C. Kobayashi, R. Suzuki, K. Miyamoto, B. Hancock, C. Dibden, and J. S. Edmonds, Nuclear Instruments and Methods in Physics Research Section B: Beam Interactions with Materials and Atoms 223-224, 116 (2004), proceedings of the Ninth International Conference on Accelerator Mass Spectrometry.

${ }^{9}$ G. D. Alton, Review of Scientific Instruments 65, 1141 (1994).

${ }^{10}$ E. Speth, H. Falter, P. Franzen, U. Fantz, M. Bandyopadhyay, S. Christ, A. Encheva, M. Früschle, D. Holtum, B. Heinemann, W. Kraus, A. Lorenz, C. Martens, P. McNeely, S. Obermayer, R. Riedl, R. Süss, A. Tanga, R. Wilhelm, and D. Wünderlich, Nuclear Fusion 46, S220 (2006).

${ }^{11}$ G. Fubiani, R. S. Hemsworth, H. P. L. de Esch, and L. Svensson, Phys. Rev. ST Accel. Beams 12, 050102 (2009).

${ }^{12}$ U. Fantz, C. Hopf, R. Friedl, S. Cristofaro, B. Heinemann, S. Lishev, and A. Mimo, Fusion Engineering and Design 136, 340 (2018), special Issue: Proceedings of the 13th International Symposium on Fusion Nuclear Technology (ISFNT-13).

${ }^{13}$ G. Cartry, D. Kogut, K. Achkasov, J.-M. Layet, T. Farley, A. Gicquel, J. Achard, O. Brinza, T. Bieber, H. Khemliche, P. Roncin, and A. Simonin, New Journal of Physics 19, 025010 (2017).

${ }^{14}$ I. Furno, R. Agnello, U. Fantz, A. Howling, R. Jacquier, C. Marini, G. Plyushchev, P. Guittienne, and A. Simonin, EPJ Web Conf. 157, 03014 (2017).

${ }^{15}$ C. Marini, R. Agnello, B. Duval, I. Furno, A. Howling, R. Jacquier, A. Karpushov, G. Plyushchev, K. Verhaegh, P. Guittienne, U. Fantz, D. Wünderlich, S. Béchu, and A. Simonin, Nuclear Fusion 57, 036024 (2017).

${ }^{16}$ R. Agnello, S. Béchu, I. Furno, P. Guittienne, A. Howling, R. Jacquier, G. Plyushchev, M. Barbisan, R. Pasqualotto, I. Morgal, and A. Simonin, Nuclear Fusion 60, 026007 (2019).

${ }^{17} \mathrm{C}$. Wimmer and U. Fantz, AIP Conference Proceedings 1655 , 040004 (2015).

${ }^{18}$ G. Fubiani, L. Garrigues, G. Hagelaar, N. Kohen, and J. P. Boeuf, New Journal of Physics 19, 015002 (2017).

${ }^{19}$ M. A. Lieberman and A. J. Lichtenberg, Principles of Plasma Discharges and Materials Processing (Wiley Interscience, 2005).

${ }^{20}$ Y. Itikawa, Atomic Data and Nuclear Data Tables 14, 1 (1974).

${ }^{21}$ R. K. Janev, Atomic and Molecular Processes in Fusion Edge Plasmas (Springer, 1995).

${ }^{22}$ J. F. Williams, Journal of Physics B: Atomic and Molecular Physics 8, 1683 (1975).

${ }^{23}$ J. F. Williams, Journal of Physics B: Atomic and Molecular Physics 8, 2191 (1975).

${ }^{24}$ I. Bray, D. A. Konovalov, and I. E. McCarthy, Phys. Rev. A 44, 5586 (1991)

${ }^{25}$ R. K. Janev, D. Reiter, and U. Samm, "Collision processes in low-temperature hydrogen plasmas," Tech. Rep. 4105 (FZJuelich, 2003).

${ }^{26}$ S. J. Buckman and A. V. Phelps, The Journal of Chemical Physics 82, 4999 (1985).

${ }^{27}$ J.-S. Yoon, M.-Y. Song, J.-M. Han, S. H. Hwang, W.-S. Chang, B. Lee, and Y. Itikawa, Journal of Physical and Chemical Ref- erence Data 37, 913 (2008).

${ }^{28}$ J. P. England, M. T. Elford, and R. W. Crompton, Australian Journal of Physics 41, 573 (1988).

${ }^{29}$ D. K. Gibson, Australian Journal of Physics 23, 683 (1970).

${ }^{30}$ M. G. Heaps and A. E. S. Green, Journal of Applied Physics 46, 4718 (1975).

${ }^{31}$ H. Ehrhardt, L. Langhans, F. Linder, and H. S. Taylor, Phys. Rev. 173, 222 (1968).

${ }^{32}$ R. H. Garvey, H. S. Porter, and A. E. S. Green, Journal of Applied Physics 48, 4353 (1977).

${ }^{33}$ R. K. Janev, Elementary Processes in Hydrogen-Helium Plasmas (Springer, 1987).

${ }^{34}$ G. Fubiani and J. P. Boeuf, Physics of Plasmas 20, 113511 (2013).

${ }^{35} \mathrm{~K}$. Nanbu, Plasma Science, IEEE Transactions on 28, 971 (2000).

${ }^{36}$ V. V. Serikov, S. Kawamoto, and K. Nanbu, Plasma Science, IEEE Transactions on 27, 1389 (1999).

${ }^{37}$ S. Kolev, G. J. M. Hagelaar, and J. P. Boeuf, Physics of Plasmas 16, 042318 (2009).

${ }^{38}$ J. P. Boeuf, G. J. M. Hagelaar, P. Sarrailh, G. Fubiani, and N. Kohen, Plasma Sources Science and Technology 20, 015002 (2011).

${ }^{39}$ F. Gaboriau and J. P. Boeuf, Plasma Sources Science and Technology 23, 065032 (2014).

${ }^{40}$ P. S. Krstić, Phys. Rev. A 66, 042717 (2002).

${ }^{41}$ P. S. Krstić, Nuclear Instruments and Methods in Physics Research Section B: Beam Interactions with Materials and Atoms 241, 58 (2005).

${ }^{42}$ A. V. Phelps, Journal of Physical and Chemical Reference Data 19, 653 (1990).

${ }^{43}$ A. V. Phelps, Phys. Rev. E 79, 066401 (2009).

${ }^{44}$ P. S. Krstić and D. R. Schultz, Journal of Physics B: Atomic, Molecular and Optical Physics 36, 385 (2003).

${ }^{45}$ P. S. Krstic and D. R. Schultz, Atomic and Plasma-Material Interaction Data for Fusion 8, 1 (1998).

${ }^{46} \mathrm{C} . \mathrm{F}$. Barnett, "Collisions of $\mathrm{h}, \mathrm{h} 2$, he, and li atoms and ions with atoms and molecules," Tech. Rep. ORNL-6086 (Oak ridge national laboratory, 1990).

${ }^{47}$ V. E. Golant, A. P. Zhilinsky, and I. E. Sakharov, Fundamentals of plasma physics (John Wiley \& Sons, 1980).

${ }^{48} \mathrm{D}$. Nicholson, Introduction to plasma theory (John Wiley \& Sons, 1983).

${ }^{49}$ R. Agnello, M. Barbisan, I. Furno, P. Guittienne, A. A. Howling, R. Jacquier, R. Pasqualotto, G. Plyushchev, Y. Andrebe, S. Béchu, I. Morgal, and A. Simonin, Review of Scientific Instruments 89, 103504 (2018).

${ }^{50}$ D. S. Thompson, R. Agnello, I. Furno, A. Howling, R. Jacquier, G. Plyushchev, and E. E. Scime, Physics of Plasmas 24, 063517 (2017).

${ }^{51} \mathrm{R}$. Futtersack, Modélisation fluide du transport magnétisé dans les plasmas froids, Ph.D. thesis, Université Toulouse III Paul Sabatier (2014).

${ }^{52}$ C. Rebont, N. Claire, T. Pierre, and F. Doveil, Phys. Rev. Lett. 106, 225006 (2011).

${ }^{53}$ H. Tanaka, N. Ohno, Y. Tsuji, K. Okazaki, and S. Kajita, Contributions to Plasma Physics 52, 424 (2012).

${ }^{54}$ L. Popelier, A. Aanesland, and P. Chabert, J. Phys. D: Appl. Phys. 44, 315203 (2011). 